\title{
Mehr als nur ein Schlagwort
}

\author{
Partizipation bzw. Inklusion am Beispiel der Werkstättensprecher der Diakonie de La Tour
}

Rund 2.000 Menschen werden in den Einrichtungen der Diakonie de La Tour betreut, gepflegt und gefördert. Unter ihnen auch viele Menschen mit Behinderung. In Beschäftigungswerkstätten gehen sie Tagfür Tag einer Arbeit nach. Wie alle Menschen haben auch sie im beruflichen Alltag Probleme, die es zu besprechen gilt. Mit dem System der Werkstättensprecher erhalten sie Vertreter aus eigenen Reihen, die für ihre Anliegen eintreten - aktive Mitbestimmung im alltäglichen Leben wird für die Klienten somit möglich.

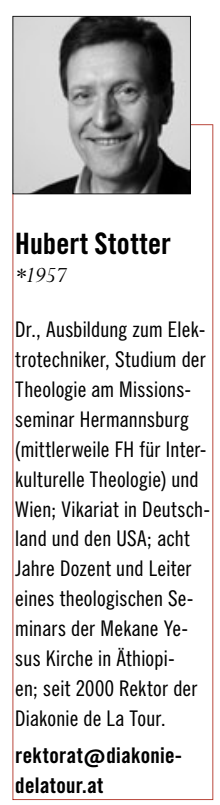

Jeder Mensch ist eine von Gott geschaffene, einzigartige Persönlichkeit - so der Grundsatz christlichen Glaubens. Davon ausgehend wäre es selbstverständlich, dass auch jeder Mensch ein eigenverantwortliches, selbstbestimmtes Leben führen kann. Was aber, wenn ein Mensch nicht der Norm entspricht - mit einer Behinderung lebt und von rechtlicher Seite her mit einem Sachwalter betraut ist? Bedeutet ein Vormund gleichzeitig auch, bevormundet zu werden?

\section{Grundrecht menschlicher Existenz}

Partizipation bzw. Inklusion sind heute keine bloßen Schlagwörter mehr. Sie beschreiben, dass die interaktive Teilhabe am gesellschaftlichen Leben als Grundrecht gesehen wird und wesentlicher Bestandteil menschlicher Existenz ist. Dennoch brauchte es auch in der Diakonie mancherorts lange, bis sich gewisse Ansichten änderten und Partizipation bzw. Inklusion zu Grundprinzipien in der Begleitung von Menschen mit Assistenzbedarf entwickelten. Das dürfte auch in einer zu stark ausgeprägten Fürsorgehaltung begründet gewesen sein, welche dem christlichen Ansatz der Nächstenliebe ja naheliegt. So traten beispielsweise in unseren Werkstätten für Menschen mit Assistenzbedarf erst mit deutlicher Verzögerung Entwicklungen in Richtung Partizipation ein, obwohl diese im gesellschaftlichen Umfeld längst selbstverständlich waren. Denn während es in den meisten Unternehmen eine Mitarbeitervertretung wie Betriebsrat und Gewerkschaft gab, konnten Menschen mit Assistenzbedarf in vielen Einrichtungen der Diakonie lange Zeit nicht von solchen Dingen profitieren.

\section{Das Modell der Werkstättensprecher}

Um Partizipation bzw. Inklusion möglich zu machen, wurden in der Diakonie de La Tour schließlich Modelle wie das der Werkstät- tensprecher eingeführt. Mittels schriftlicher Wahl oder Handzeichen werden dabei im Rahmen von Klientenbesprechungen Vertreter gewählt. Dabei kommt auch die Unterstützte Kommunikation zum Einsatz: Im Vorfeld mit Fotos aufbereitete Wahlformulare ermöglichen für die Klienten leichte Verständlichkeit. Als Vertreter von rund 200 Klienten fungieren die Sprecher - derzeit sind zehn aktiv - als erste Ansprechpartner für berufliche Alltagsprobleme. Gemeinsam mit Mitarbeitern moderieren sie die einmal im Monat stattfindenden Klientenbesprechungen. Im Teamwork erstellte Protokolle der Sitzungen werden an die jeweilige Einrichtungsleitung sowie Abteilungsleitung versandt. Werkstättensprecher vertreten die Interessen der Klienten und bringen ihre Anliegen bei den entsprechenden Stellen ein. Häufig betreffen die Themen Wünsche der Klienten bezüglich Ausflügen, Veranstaltungen etc., aber auch konkrete Hinweise und Anregungen, wie zum Beispiel die barrierefreie Gestaltung des Seezuganges oder die Beschattung der Liegewiese (die Diakonie de La Tour verfügt über ein Grundstück am Ossiacher See) werden angeregt. Darüber hinaus gibt es eine standortbezogene Vernetzung, gelegentlich auch standortübergreifende Treffen und Austauschmöglichkeiten.

\section{Aktive Mitbestimmung}

Die Werkstättensprecher haben auch das Recht, bei bestimmten Themen, die Klienten betreffen, an den Teamsitzungen der Mitarbeiter teilzunehmen. Auf diese Weise wurden beispielsweise bei der Planung einer neuen Beschäftigungswerkstätte die Wünsche der Klienten in Bezug auf Räumlichkeiten und Ausstattung bei der Planung miteinbezogen. Zudem erhalten Klientenvertreter die Gelegenheit, an Treffen übergeordneter Netzwerke wie des ÖAR (Österreichische Arbeitsgemeinschaft für Rehabilitation) in Wien oder anderer Gruppen teilzunehmen. Klient Jürgen Ceplak ist beispielsweise Mitglied des Netzwerkes „Expertentreffen“. Veranstaltungen der Gruppe besucht er mit einer Begleitperson. Gemeinsam verfassen sie ein Protokoll im Format „Leicht Lesen“, das dann auch den anderen Klientenvertretern zur Verfügung gestellt wird.

Abstract / Das Wichtigste in Kürze Was bedeutet Partizipation bzw. Inklusion für Menschen mit Behinderung? Wie können sie ihre Rechte und Wünsche geltend machen?

Keywords / Stichworte Partizipation, Inklusion 